\title{
Atrioventricular dissociation between two atrioventricular nodal rhythms
}

\author{
R. Bernard, H. Vainsel, and L. Schamroth \\ From Groupement Scientifique pour le Diagnostic et le Traitement de Cardiopathies, \\ Clinique Cardiologique - Département de Cardiologie, Hôpital Universitaire St. Pierre, \\ Bruxelles, Belgium; and Baragwanath Hospital and the University of the Witwatersrand, \\ fohannesburg, S.A.
}

A case presenting with AV nodal escape rhythms after obvious sinus depression is described. The escape rhythms arise from two dissociated AV nodal pacemakers with differential physiological properties. Variation in the rate of the upper AV nodal pacemaker results in a form of escape-capture bigeminy. Temporary acceleration of the sinus rhythm results in dissociation between sinus and lower AV nodal pacemakers, or conducted sinus rhythm.

AV dissociation usually occurs between sinus or, less commonly, an ectopic atrial rhythm, and an AV nodal or a ventricular rhythm. Dissociation between two AV nodal pacemakers is exceptionally rare; only a few cases have been described (Luten and Jensen, 1932; Schott, 1937; Miller and Sharrett, 1957; Castellanos, Azan, and Calviño, 1958; Sanghvi, 196I; Chung, Walsh, and Massie, 1964). The following is another example of this unusual arrhythmia.

\section{Case report}

The electrocardiogram was recorded from a 35year-old woman with a moderate degree of mitral insufficiency. The blood pressure was 150/90 mmHg. She was not on digitalis therapy.

Fig. I is a continuous strip of lead VI (only the lower section has been analysed) and representative complexes from standard leads I, II, and III. The $P$ wave in lead VI is dominantly upright with a small initial negative component. The $P$ wave is slightly positive (almost equiphasic) in standard lead I, and negative in standard leads II and III. The mean manifest frontal plane $\mathbf{P}^{\prime}$ wave axis is directed at minus 80 degrees. These features reflect the classic $\mathbf{P}^{\prime}$ wave that is usually associated with retrograde atrial activation by AV nodal or ventricular impulses. This AV nodal rhythm will be referred to as the upper $A V$ nodal rhythm. The $P^{\prime} P^{\prime}$ intervals range from $I \cdot 18$ sec to $I \cdot 42 \mathrm{sec}$ (mean: $\mathrm{I} \cdot 30 \mathrm{sec}$ ), representing an $\mathrm{AV}$ nodal rate of 43 to $5 \mathrm{r}$ beats a minute (mean: 46 a minute). The $\mathbf{P}^{\prime}$ waves are dissociated from the QRS complexes. These dissociated QRS complexes are normal in configuration. Normal QRS complexes which are dissociated from the atrial deflections, and which have a contour identical to the QRS complexes of conducted sinus beats (see below), must also represent an AV nodal rhythm. This rhythm must arise from the lower regions of the AV node or junction, and will be referred to as the lower $A V$ nodal rhythm.

The RR intervals of the lower AV nodal rhythm range from $I \cdot I 8 \mathrm{sec}$ to $\mathrm{I} \cdot 28 \mathrm{sec}$ (mean: $\mathrm{I} \cdot 24 \mathrm{sec}$ ), representing a rate range of 47 to $5 \mathrm{I}$ beats a minute (mean: 49 beats a minute), a mean rate which is slightly faster than that of the upper AV nodal rhythm. The upper and lower AV nodal impulses interfere with each other's progress within the AV node. This is because the upper AV nodal impulse usually encounters lower AV nodal refractoriness consequent to prior discharge or activation by the lower AV nodal pacemaker. This results in AV dissociation between the two AV nodal pacemakers. Since the upper AV nodal rhythm is slightly slower than the lower AV nodal rhythm, the $P^{\prime}$ waves occur progressively later in relation to the $Q R S$ complexes. When the upper AV nodal impulse occurs relatively late after the lower AV nodal impulse, it encounters a responsive lower AV node or junction, and is consequently conducted to, momentarily activating or 'capturing', the ventricles. This constitutes a ventricular capture beat. The capturing impulse, because of its prematurity, is conducted with aberration, resulting in a right bundle-branch block pattern.

Fig. 2 (a continuous strip of lead VI) is a later section of the same recording, and shows escapecapture bigeminy. This is reflected in the upper strip by the first Io QRS complexes which are rhythmically spaced as 5 bigeminal couplets. Each bigeminal couplet is constituted by an AV nodal escape beat (from the lower AV nodal pacemaker) followed by a ventricular capture which is effected by an impulse from the upper AV nodal pacemaker. The period of escape-capture bigeminy is followed consecutively by: (a) two further escape beats which are dissociated from the concomitant $\mathbf{P}^{\prime}$ waves; (b) a further escape-capture bigeminal couplet; (c) another escape beat with AV dissociation, and (d) four lower AV nodel escape beats with AV dissociation from sinus rhythm. It is 


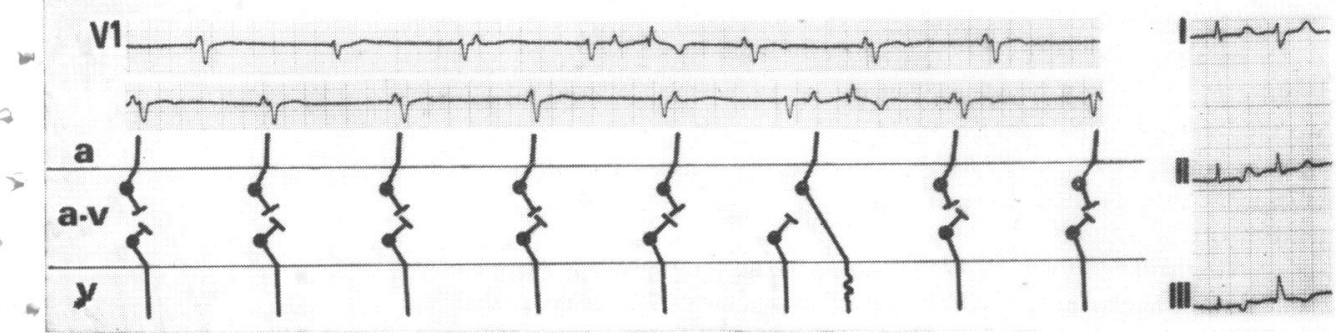

FIG. I Electrocardiogram: a continuous strip of lead VI and representative complexes from standard leads I, II, and III showing $A V$ dissociation between $A V$ nodal pacemakers.

important to note that the configuration of a normal sinus $P$ wave in lead VI is usually biphasic

$\checkmark$ with a small initial positivity and a prominent terminal negativity. In this case, the positive component is absent, and the negative component is exaggerated - an expression of left atrial enlargement due to the mitral insufficiency.

The next four beats (the first four beats in the third strip) reflect further escape with AV dissociation between (a) the upper and lower AV

- nodal impulses (first two beats, though, no definite atrial deflection can be discerned) or, (b) between sinus and lower AV nodal impulses (third and fourth beats). The second four beats of the third strip reflect conducted sinus rhythm. The PR interval measures $0.18 \mathrm{sec}$. The $P P$ intervals range from $\mathrm{I} \cdot 04 \mathrm{sec}$ to $\mathrm{I} \cdot \mathrm{I} 2 \mathrm{sec}$, representing a rate of 54 to 58 beats a minute, with progressive slowing of the sinus discharge until it fails to manifest. The bottom strip reflects: AV dissociation between upper and lower AV nodal rhythms - the first and fifth beats; escape-capture bigeminy - the second and third beats, and the last two couplets; and AV dissociation between sinus and lower AV nodal pacemakers - the fourth beat. The $P^{\prime}$ wave associated with the first bigeminal couplet in this strip has a configuration that is between that of "the 'pure' sinus $P$ wave, and the 'pure' upper AV nodal $P^{\prime}$ wave, and therefore represents an atrial fusion beat.

\section{Discussion}

The AV nodal rhythms are idionodal - escape - rhythms which come about because of obvious depression or slowing of the sinus rhythm, which may, in addition, be complicated by SA block (see below). The sinus rhythm must be slowed so that its cycle exceeds the upper AV nodal cycle, thereby permitting the upper AV nodal rhythm to usurp control of the atria.

When the sinus rate temporarily accelerates - an expression of the acceleration phase of a marked sinus arrhythmia - the following manifestations result.

A) The sinus rhythm is dissociated from the lower AV nodal rhythm, e.g. the second strip of Fig. 2. This occurs when the sinus rate just exceeds the upper AV nodal rate, and approximates the lower AV nodal rate.

B) The sinus impulses usurp complete control of the heart, i.e. the atria as well as the ventricles, resulting in conducted sinus rhythm, e.g. in the third strip of Fig. 2. This occurs when the sinus rate exceeds both the upper and lower AV nodal rates.

FIG. 2 Electrocardiogram: a continuous strip of lead VI showing escape-capture bigeminy between upper and lower $A V$ nodal pace-makers, and dissociation between sinus and/or upper $A V$ nodal pacemakers and a lower $A V$ nodal pacemaker.

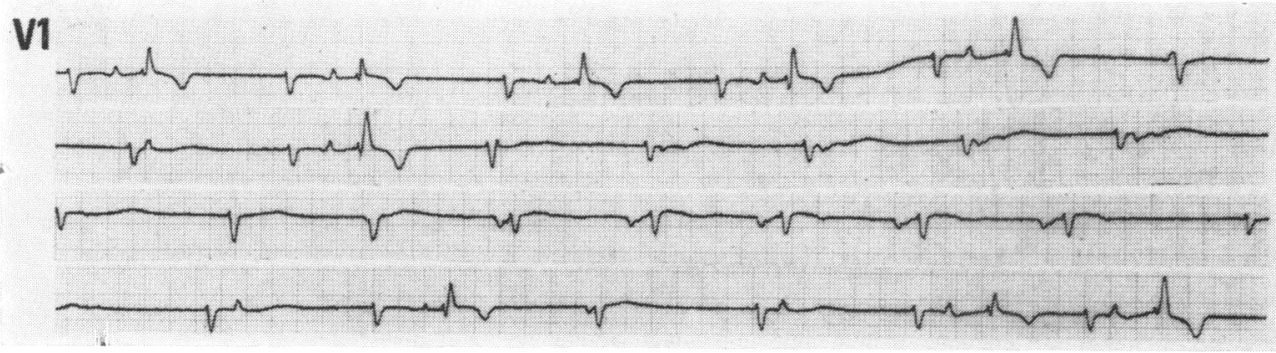


The escape-capture bigeminy is unusual in that it consists of the escape of a lower AV nodal pacemaker followed by a ventricular capture which is effected by an upper AV nodal pacemaker. Most cases of escape-capture bigeminy are due to capture by sinus impulses. The rhythm is possible because of an obvious increase in the duration of the upper $\mathrm{AV}$ nodal cycle to $\mathrm{I} \cdot 70 \mathrm{sec}$, i.e. an upper AV nodal slowing to a rate of 35 beats a minute, a slowing that results in a conspicuous discrepancy between the cycles of the upper and lower AV nodal rhythms. For the mechanism of escape-capture bigeminy to occur, the effective upper cycle - the cycle between the upper impulses which reach the level of the lower pacemaker - must be so prolonged, that it exceeds the sum of the lower pacemaker cycle and the ensuing refractory period (Schamroth and Dubb, 1965). Furthermore, it is also evident that the potential sinus cycle during the period of escape-capture bigeminy must be longer than $\mathrm{I} \cdot 70 \mathrm{sec}$, for otherwise the sinus rhythm would become manifest. The potential sinus rhythm must therefore be less than 35 beats a minute. A slow potential sinus rhythm of this magnitude may represent an obvious sinus bradycardia, or, perhaps more likely, a sinus rhythm complicated by SA block. It is further evident that the depression of automaticity, which affects the sinus pacemaker during the period of escape-capture bigeminy, also affects the upper AV nodal pacemaker, since the AV nodal rates of 35 beats a minute are slower than the inherent idionodal rhythm which is usually about 50 beats a minute. Moreover, the depressing effect during this period is most obvious on the upper AV nodal rhythm with little influence on the lower AV nodal rhythm, thereby reflecting differential physiological properties of these pacemakers.

We are indebted to the Photographic Department, Department of Medicine, University of the Witwatersrand, for the photographic reproductions.

\section{References}

Castellanos, A., Azan, L., and Calviño, J. M. (1958). Dissociation with interference between pacemakers located within the A-V conducting system. American Heart fournal, 56, 562.

Chung, K. Y., Walsh, T. J., and Massie, E. (1964). Double A-V nodal rhythm. Fapanese Heart fournal, 5, 17 I.

Luten, D., and Jensen, J. (1932). Ventricular bigeminy (parasystole or reciprocal rhythm) in atrioventricular rhythm. American Heart fournal, 7, 593.

Miller, R., and Sharrett, R. H. (1957). Interference dissociation. Circulation, 16, 803.

Sanghvi, L. M. (I96r). Unusual cardiac arrhythmias in myocardial infarction. American fournal of Cardiology, 8, 147.

Schamroth, L., and Dubb, A. (1965). Escape-capture bigeminy; mechanisms in S-A block, A-V block, and reversed reciprocal rhythm. British Heart fournal, 27, 667.

Schott, A. (1937). Atrioventricular rhythm with and without retrograde block. American Heart fournal, r3, 6r.

Requests for reprints to Dr. L. Schamroth, I55 Barry Hertzog Avenue, Emmarentia, Johannesburg, South Africa. 